\title{
DISCOVERY OF VARIABILITY IN THE VERY HIGH ENERGY $\gamma$-RAY EMISSION OF 1ES 1218+304 WITH
} VERITAS

\author{
V. A. Acciari ${ }^{1}$, E. Aliu ${ }^{2}$, M. Beilicke ${ }^{3}$, W. Benbow ${ }^{1}$, D. Boltuch ${ }^{4}$, M. Böttcher $^{5}$, S. M. Bradbury $^{6}$, V. Bugaev $^{3}$, \\ K. Byrum ${ }^{7}$, A. Cesarini ${ }^{8}$, L. Ciupik ${ }^{9}$, P. Cogan ${ }^{10}$, W. Cui ${ }^{11}$, R. Dickherber ${ }^{3}$, C. Duke ${ }^{12}$, A. Falcone ${ }^{13}$, J. P. Finley ${ }^{11}$, \\ G. Finnegan $^{14}$, L. Fortson ${ }^{9}$, A. Furniss ${ }^{15}$, N. Galante ${ }^{1}$, D. Gall ${ }^{11}$, K. Gibbs ${ }^{1}$, R. Guenette ${ }^{10}$, G. H. Gillanders ${ }^{8}$,

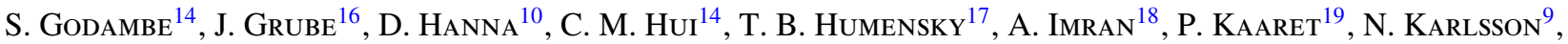

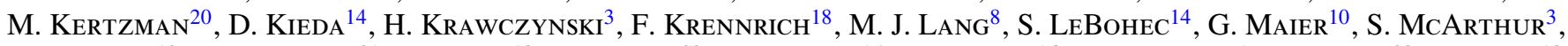 \\ A. McCann ${ }^{10}$, P. Moriarty ${ }^{21}$, T. Nagai ${ }^{18}$, R. A. Ong ${ }^{22}$, A. N. Otte ${ }^{15}$, D. PAndel ${ }^{16}$, J. S. Perkins ${ }^{1}$, A. PiChel $^{23}$, M. Pohl $^{18}$, \\ J. QuinN ${ }^{16}$, K. Ragan ${ }^{10}$, L. C. Reyes ${ }^{24}$, P. T. ReYNOLds ${ }^{25}$, E. RoAChe ${ }^{1}$, H. J. Rose ${ }^{6}$, M. SchroedteR ${ }^{18}$, G. H. SEMBroski ${ }^{11}$, \\ A. W. Smith ${ }^{7}$, D. Steele ${ }^{9}$, S. P. Swordy ${ }^{17}$, M. Theiling ${ }^{1}$, S. Thibadeau ${ }^{3}$, V. V. VASsiliev ${ }^{22}$, S. Vincent $^{14}$, S. P. WAKely $^{17}$, \\ T. C. WeEkes ${ }^{1}$, A. Weinstein ${ }^{22}$, T. Weisgarber ${ }^{17}$, AND D. A. Williams ${ }^{15}$ \\ ${ }^{1}$ Fred Lawrence Whipple Observatory, Harvard-Smithsonian Center for Astrophysics, Amado, AZ 85645, USA \\ ${ }^{2}$ Department of Physics and Astronomy, Barnard College, Columbia University, NY 10027, USA \\ ${ }^{3}$ Department of Physics, Washington University, St. Louis, MO 63130, USA \\ ${ }^{4}$ Department of Physics and Astronomy and the Bartol Research Institute, University of Delaware, Newark, DE 19716, USA \\ ${ }^{5}$ Astrophysical Institute, Department of Physics and Astronomy, Ohio University, Athens, OH 45701, USA \\ ${ }^{6}$ School of Physics and Astronomy, University of Leeds, Leeds, LS2 9JT, UK \\ ${ }^{7}$ Argonne National Laboratory, 9700 S. Cass Avenue, Argonne, IL 60439, USA \\ ${ }^{8}$ School of Physics, National University of Ireland Galway, University Road, Galway, Ireland \\ ${ }^{9}$ Astronomy Department, Adler Planetarium and Astronomy Museum, Chicago, IL 60605, USA \\ ${ }^{10}$ Physics Department, McGill University, Montreal, QC H3A 2T8, Canada \\ ${ }^{11}$ Department of Physics, Purdue University, West Lafayette, IN 47907, USA \\ ${ }^{12}$ Department of Physics, Grinnell College, Grinnell, IA 50112-1690, USA \\ ${ }^{13}$ Department of Astronomy and Astrophysics, 525 Davey Lab, Pennsylvania State University, University Park, PA 16802, USA \\ ${ }^{14}$ Department of Physics and Astronomy, University of Utah, Salt Lake City, UT 84112, USA \\ ${ }^{15}$ Santa Cruz Institute for Particle Physics and Department of Physics, University of California, Santa Cruz, CA 95064, USA \\ ${ }^{16}$ School of Physics, University College Dublin, Belfield, Dublin 4, Ireland \\ ${ }^{17}$ Enrico Fermi Institute, University of Chicago, Chicago, IL 60637, USA \\ ${ }^{18}$ Department of Physics and Astronomy, Iowa State University, Ames, IA 50011, USA; imranisu@ @iastate.edu \\ ${ }^{19}$ Department of Physics and Astronomy, University of Iowa, Van Allen Hall, Iowa City, IA 52242, USA \\ ${ }^{20}$ Department of Physics and Astronomy, DePauw University, Greencastle, IN 46135-0037, USA \\ ${ }^{21}$ Department of Life and Physical Sciences, Galway-Mayo Institute of Technology, Dublin Road, Galway, Ireland \\ ${ }^{22}$ Department of Physics and Astronomy, University of California, Los Angeles, CA 90095, USA \\ ${ }^{23}$ Instituto de Astronomia y Fisica del Espacio, Casilla de Correo 67-Sucursal 28, (C1428ZAA) Ciudad Autónoma de Buenos Aires, Argentina \\ ${ }^{24}$ Kavli Institute for Cosmological Physics, University of Chicago, Chicago, IL 60637, USA \\ ${ }^{25}$ Department of Applied Physics and Instrumentation, Cork Institute of Technology, Bishopstown, Cork, Ireland \\ Received 2009 November 18; accepted 2009 December 23; published 2010 January 14
}

\begin{abstract}
We present results from an intensive VERITAS monitoring campaign of the high-frequency peaked BL Lac object 1ES $1218+304$ in 2008/2009. Although 1ES 1218+304 was detected previously by MAGIC and VERITAS at a persistent level of $\sim 6 \%$ of the Crab Nebula flux, the new VERITAS data reveal a prominent flare reaching 20\% of the Crab. While very high energy (VHE) flares are quite common in many nearby blazars, the case of $1 \mathrm{ES} 1218+304$ (redshift $z=0.182$ ) is particularly interesting since it belongs to a group of blazars that exhibit unusually hard VHE spectra considering their redshifts. When correcting the measured spectra for absorption by the extragalactic background light, 1ES $1218+304$ and a number of other blazars are found to have differential photon indices $\Gamma \leqslant$ 1.5. The difficulty in modeling these hard spectral energy distributions in blazar jets has led to a range of theoretical $\gamma$-ray emission scenarios, one of which is strongly constrained by these new VERITAS observations. We consider the implications of the observed light curve of 1ES 1218+304, which shows day scale flux variations, for shock acceleration scenarios in relativistic jets, and in particular for the viability of kiloparsec-scale jet emission scenarios.
\end{abstract}

Key words: BL Lacertae objects: individual (1ES 1218+304 = VER J1221+301) - galaxies: active - gamma rays: galaxies

Online-only material: color figures

\section{INTRODUCTION}

The very high energy (VHE) $\gamma$-ray sky has seen a dramatic increase in the number of sources, with 26 detected active galactic nuclei ${ }^{26}$ (AGNs) over redshifts of 0.0018-0.536. All, except two, are blazars with their jets closely aligned to our line of sight. Blazars as a class are comprised of BL Lacertae

\footnotetext{
$\overline{26}$ For updates, see http://tevcat.uchicago.edu.
}

objects, optically violent variables and other radio-loud quasars (Urry \& Padovani 1995). Nonthermal emission dominates the broadband continuum spectra of blazars.

The characteristic double-peaked spectral energy distribution of blazars is usually attributed to synchrotron radiation in the radio to X-ray wavebands, and inverse-Compton upscattering of soft target photons by relativistic electrons at $\mathrm{GeV}$ and $\mathrm{TeV}$ energies (Maraschi et al. 1992; Bloom \& Marscher 1996). 
Alternative emission scenarios involve hadronic interactions producing neutral pions which decay into photons (Mannheim $\&$ Bierman 1992) and proton synchrotron radiation (Aharonian 2000). The latter models provide a link between AGNs and possible sources of ultra-high-energy cosmic rays. A better understanding of the underlying acceleration and emission processes requires a larger sample of sources, which is now being provided by the Fermi Gamma-ray Space Telescope (Fermi) at $0.1 \mathrm{GeV}$ to hundreds of $\mathrm{GeV}$ and by the imaging atmospheric Cherenkov telescopes (IACT), namely the MAGIC, H.E.S.S., and VERITAS Observatories, that provide coverage primarily in the $100 \mathrm{GeV}-10 \mathrm{TeV}$ regime.

To date, blazars detected at VHE energies are predominantly high-frequency peaked BL Lacertae objects (HBLs). Nevertheless, a few objects with lower peak energies, namely low-frequency-peaked BL Lacertae (LBLs; Albert et al. 2007; Teshima et al. 2008) and intermediate-frequency-peaked BL Lacertae objects (IBLs; see Acciari et al. 2008a, 2008b, 2009b; Ong et al. 2009) were detected recently by IACTs at TeV energies. The $\gamma$-ray luminosity of LBLs and IBLs generally peaks at sub-GeV to tens of $\mathrm{GeV}$ in energy, and they are therefore easily detectable by Fermi while their detectability in the $\mathrm{TeV}$ regime by IACTs is more difficult due to their soft VHE spectra.

Among the HBLs, which are typically the domain of the TeV telescopes, a number of objects exhibit unusually hard intrinsic power-law energy spectra $\left(d N / d E \sim E^{-\Gamma_{i}}\right)$ after correcting for the $\gamma \gamma \rightarrow e^{+} e^{-}$absorption by the cosmological diffuse EBL radiation field. While the measured spectral indices, $\Gamma_{m}$, of these blazars (1ES 1101-232, 1ES 0347-121, 1ES 0229+200, 1ES 1218+304, RGB J0710+591) range from 2.5 to 3.1 (see Aharonian et al. 2006a, 2007a, 2007b; Albert et al. 2006; Acciari et al. 2009a; Perkins et al. 2009), the absorption-corrected spectral indices suggest very hard intrinsic source spectra in the VHE regime with $\Gamma_{i} \leqslant 1.28 \pm 0.28$ (Krennrich et al. 2008), based on recent lower limits to the EBL from galaxy counts (Levenson \& Wright 2008).

Similar hard energy spectra are found by Fermi, where, for example, the measured spectral indices for RGB J0710+591 and 1ES $1440+122$ are found to be $\Gamma=1.21 \pm 0.25$ and $1.18 \pm 0.27$ over energies from $0.2 \mathrm{GeV}$ to tens of $\mathrm{GeV}$ (Abdo et al. 2009a). While the findings of very hard VHE spectra are based on EBL constraints from galaxy counts, the Fermi spectra directly resemble the intrinsic source spectra since absorption by the EBL is minimal for the covered energy range. The photon spectrum of 1ES 1218+304 measured by Fermi yields a spectral index of $\Gamma=1.63 \pm 0.12$ between $0.2 \mathrm{GeV}$ and $\sim 300 \mathrm{GeV}$, but absorption may already play a role at the high-energy end (Abdo et al. 2009a).

Diffusive shock-acceleration theory (for a review, see Malkov \& Drury 2001) generally yields a limit to the spectral index of $\mathrm{GeV}-\mathrm{TeV}$ photon spectra resulting from inverse-Compton scattering of $\Gamma_{\mathrm{i}} \geqslant 1.5$. Only recently, numerical studies by Stecker et al. (2007) indicate that sufficiently hard electron spectra could be generated by diffusive shock acceleration in relativistic shocks, for the production of photon spectra $1.0<\Gamma_{i}<1.5$. However, Böttcher et al. (2008) suggest that even for a hard-spectrum electron population, in the framework of a synchrotron self-Compton (SSC) scenario, the resulting $\mathrm{GeV}-\mathrm{TeV} \gamma$-ray spectra should experience substantial softening from Klein-Nishina effects making $\gamma$-ray spectra with $\Gamma_{i}<1.5$ less likely.

Other approaches to explain the hard $\gamma$-ray spectra are offered by ad hoc assumptions about the electron distribution, an additional absorption component in the source, or a postulate of new physics describing the propagation of $\gamma$-rays on extragalactic distance scales. Katarzyński et al. (2006) invoke a high low-energy cutoff in the electron distribution that could give the appearance of a hard $\gamma$-ray spectrum for a given energy regime. Aharonian et al. (2008) show that $\gamma \gamma$ absorption in the source due to a narrowband emission component from the AGN could lead to unusually hard VHE spectra. Finally, Sánchez-Conde et al. (2009) suggested an axion-like particle (ALP) that would distort the $\gamma$-ray spectrum through ALP/photon mixing on cosmological distances in the presence of intergalactic magnetic fields.

A more easily testable model that involves known physics was recently proposed by Böttcher et al. (2008). The authors attribute the hard photon spectra to inverse-Compton upscattering of ambient photons from the cosmic microwave background $(\mathrm{CMB})$, occurring in a kiloparsec-scale jet. In this case, a substantial fraction of the jet power is transported by hadrons to the outer regions of the jet, where it is dissipated into ultrarelativistic electrons. Inverse-Compton scattering in the KleinNishina regime is no longer the limitation at the highest energies since low-energy target photons from the CMB are abundant. The low magnetic field in the large-scale jet also avoids the overproduction of synchrotron radiation and allows Compton emission to dominate. Furthermore, the synchrotron emission in the radio to X-ray wavebands is assumed to originate on subparsec scales. A similar mechanism was previously suggested to explain the hard X-ray spectra observed from the kiloparsecscale jets of radio quasars (Tavecchio et al. 2007).

To date, all of the blazars exhibiting very hard spectra appear to emit at a baseline level that is consistent with this picture. However, it is also possible that the sensitivity of the current IACTs is the limiting factor in detecting day scale variations and that the underlying emission may contain flares. The question as to whether or not the $\gamma$-ray emission from 1ES $1218+304$ consists of flares or corresponds to a constant baseline emission level was one of the main motivations for VERITAS to monitor this object over a period of 5 months in the 2008/2009 season. The other motivation for deep exposures of relatively large redshift $(z=0.1-0.2)$ blazars at $\mathrm{TeV}$ energies is to provide better constraints on the EBL spectrum. The observed VHE photon spectrum can be used to constrain the EBL in the near to mid-IR. This is particularly promising for hard-spectrum blazars that provide the best sensitivity to any possible absorption feature.

\section{OBSERVATIONS AND DATA ANALYSIS}

VERITAS is an array of four $12 \mathrm{~m}$ IACTs located at the base camp of the F. L. Whipple Observatory in southern Arizona (31.68N, $110.95 \mathrm{~W}, 1.3 \mathrm{~km}$ above sea level). Each VERITAS focal plane instrument has a 3.5 field of view and consists of 499 photomultiplier tubes. The stereoscopic system has been fully operational since mid-2007 and is capable of detecting sources with a flux of $1 \%$ of the Crab Nebula in $<50 \mathrm{hr}$ of observation. In the Summer of 2009, Telescope 1 was relocated and the improved array configuration allows us to detect a point source at $1 \%$ of the Crab Nebula flux under $30 \mathrm{hr}$ of observations. The large effective area $\left(\sim 10^{5} \mathrm{~m}^{2}\right)$ of the array enables VERITAS to be sensitive over a wide range of energies (from $100 \mathrm{GeV}$ to $30 \mathrm{TeV}$ ) with an energy resolution of $15 \%-20 \%$ above $300 \mathrm{GeV}$. For further details about VERITAS, see, e.g., Holder et al. (2006). 
Table 1

Summary of Observations and Analysis of 1ES 1218+304

\begin{tabular}{lccccc}
\hline \hline Campaign & $\begin{array}{c}\text { Live Time } \\
(\mathrm{hr})\end{array}$ & $\begin{array}{c}\text { Zenith } \\
(\mathrm{deg})\end{array}$ & $\begin{array}{c}\text { Significance } \\
(\sigma)\end{array}$ & $\begin{array}{c}\Phi(>200 \mathrm{GeV}) \\
\left(10^{-12} \mathrm{~cm}^{-2} \mathrm{~s}^{-1}\right)\end{array}$ & $\begin{array}{c}\text { Units of Crab Nebula } \\
\text { Flux }(E>200 \mathrm{GeV})\end{array}$ \\
\hline $2006-2007^{\mathrm{b}}$ & 17.4 & $2-35$ & 10.4 & $12.2 \pm 2.6_{\text {stat }}$ & $0.05 \pm 0.011$ \\
$2008-2009$ & 27.2 & $2-30$ & 21.8 & $18.4 \pm 0.9_{\text {stat }}$ & $0.07 \pm 0.004$ \\
\hline
\end{tabular}

Notes. Also shown are the integral flux above $200 \mathrm{GeV}$ assuming a spectral index $\Gamma=3.07$, and the corresponding flux in units of integrated Crab Nebula flux over $200 \mathrm{GeV}$.

${ }^{a}$ Including total live time, range of zenith angles for observations, and significance of the excess events.

${ }^{b}$ See Acciari et al. (2009a).

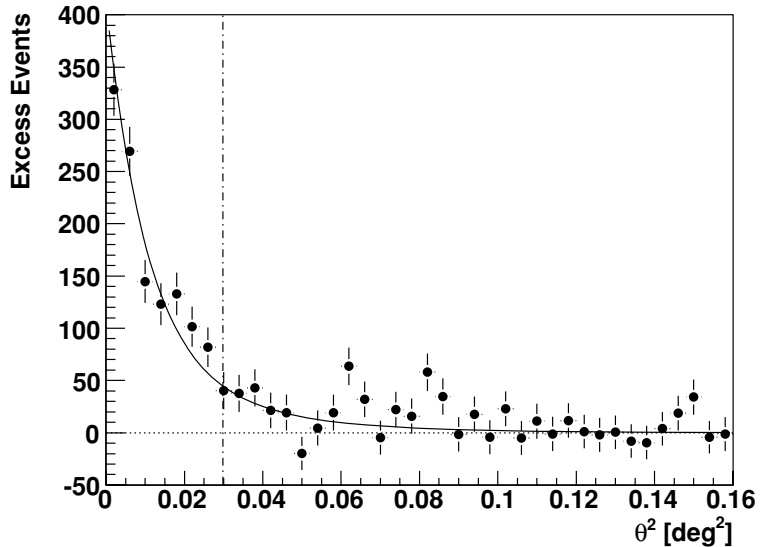

Figure 1. Distribution of $\theta^{2}$ for excess events ON-OFF from the observation of $1 \mathrm{ES} 1218+304$. The dashed-dotted line shows the boundary of the region for the spectrum cuts $\left(\theta^{2}=0.03\right)$. The solid curve indicates the expected $\theta^{2}$ distribution from a point source and provides a good fit to the data.

1ES $1218+304$ was observed by VERITAS from 2008 December to 2009 May. All data were taken in wobble mode where the source is positioned 0.5 from the center of the camera in order to allow simultaneous background estimation. A quality selection was applied to the data to reject observations affected by poor weather conditions and other anomalies, resulting in a total live time of $27.2 \mathrm{hr}$ for analysis. The mean zenith angle of the observations was $12^{\circ}$.

The data are analyzed using standard VERITAS calibration and analysis tools. Initially, the observed data set is calibrated by flat-fielding the camera gains with nightly laser runs. Following calibration and cleaning, the shower images are parameterized using a second-moment analysis (Hillas 1985). Images from individual telescopes are combined to reconstruct both arrival direction and impact position for each event. The $\gamma$-raylike events are separated from the cosmic ray background events using cuts on the mean-reduced-scaled length and meanreduced-scaled width parameters (see Aharonian et al. 2006b for a full description of the parameters). The cuts for $\gamma$-ray/hadron separation are optimized a priori for a source with a $10 \%$ Crablike flux. A circular region of radius $\theta$ is applied around the target to estimate the ON-source events. The background or OFFsource counts are estimated with off-source reflected regions (Berge et al. 2007). The significance of any excess (ON-OFF) events is calculated following Equation (17) in $\mathrm{Li} \& \mathrm{Ma}$ (1983). The angular distribution of excess events from the direction of 1ES 1218+304 (Figure 1) is comparable with the expectation from the Monte Carlo (MC) simulation of a point source.

MC simulations are used to generate a lookup table for estimating the energies of the primary $\gamma$-rays as a function of the image size and impact parameters. For spectral analysis, a looser cut (spectrum cuts) on the arrival direction of $\gamma$-rays

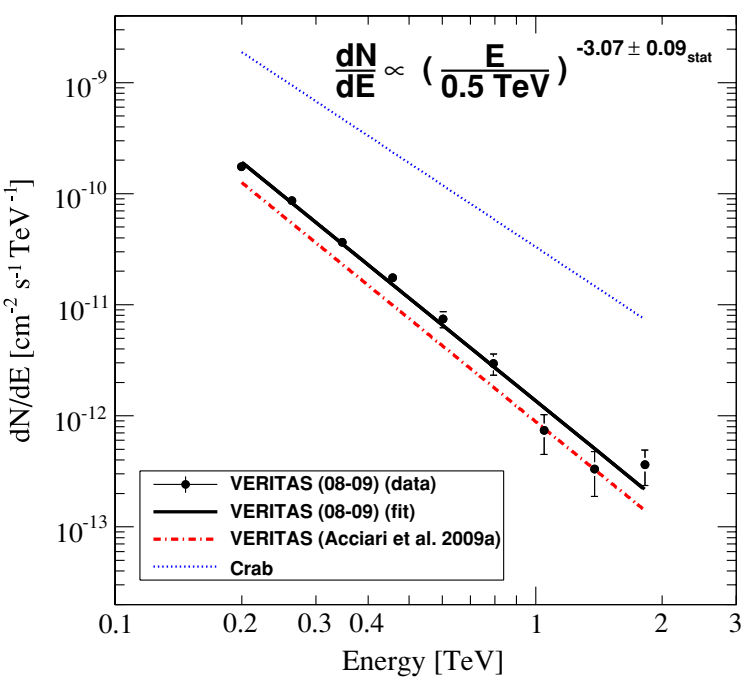

Figure 2. Observed differential energy spectrum of 1ES 1218+304 from the 2008-2009 data set. The solid line shows the power-law fit to the data (filled circles) with a spectral index value of $-3.07 \pm 0.09_{\text {stat }}$. This agrees, within statistical uncertainties, with the previous VERITAS measurement of $-3.08 \pm 0.34_{\text {stat }}$ (dashed line; Acciari et al. 2009a).

(A color version of this figure is available in the online journal.)

(a region with $\theta^{2}=0.03$ ) is applied along with three offsource reflected regions. This has the benefit of retaining a higher number of $\gamma$-ray candidates in addition to reducing the systematic uncertainties associated with the MC detector model. Both the standard cuts $\left(\theta^{2}=0.0225\right)$ and the spectrum cuts yield compatible results. Consistent results are attained with independent analysis packages.

\section{RESULTS}

Table 1 summarizes the results of the VERITAS observations of 1ES 1218+304. For the spectral analysis, we report an excess of 1155 events with a statistical significance of 21.8 standard deviations, $\sigma$, from the direction of 1ES $1218+304$ during the 2008-2009 campaign (2808 signal events, 4959 background events with a normalization of 0.33 ). Figure 2 shows the corresponding time-averaged differential energy spectrum. The spectrum extends from $200 \mathrm{GeV}$ to $1.8 \mathrm{TeV}$ and is well described $\left(\chi^{2} /\right.$ dof $\left.=8.2 / 7\right)$ by a power law,

$$
\begin{aligned}
\frac{d N}{d E}= & (11.5 \pm 0.7) \\
& \times 10^{-12}\left(\frac{E}{500 \mathrm{GeV}}\right)^{-3.07 \pm 0.09_{\text {stat }}} \mathrm{cm}^{-2} \mathrm{~s}^{-1} \mathrm{TeV}^{-1} .
\end{aligned}
$$

The errors bars are statistical only. 


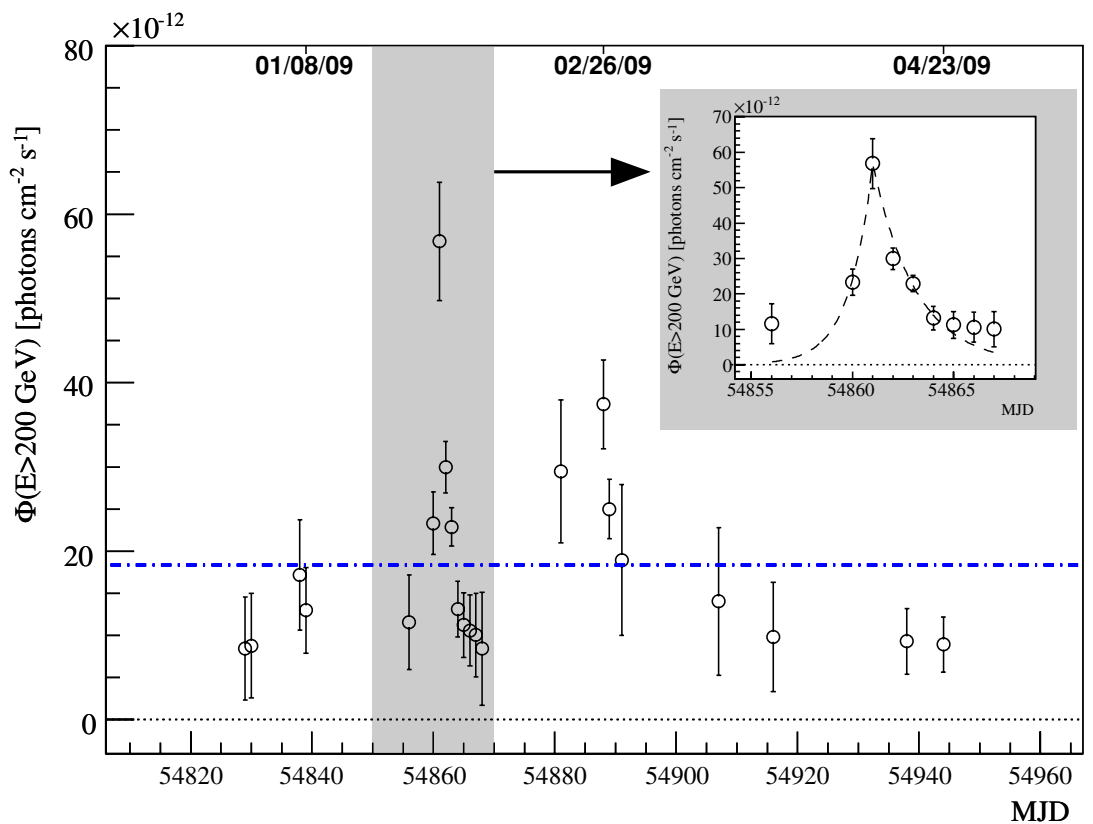

Figure 3. Night-by-night VHE light curve for 1ES 1218+304 is shown as measured from 2008 December 29 (MJD 54829) to 2009 April 23 (MJD 54944 ). The open circles represent the integral flux above $200 \mathrm{GeV}, \phi(E>200 \mathrm{GeV})$ from 1ES $1218+304$ assuming a spectral shape, $d N / d E \propto E^{-\Gamma}$ with $\Gamma=3.07$. The dashed-dotted line corresponds to the average integral flux of $\Phi(E>200 \mathrm{GeV})=\left(18.4 \pm 0.9_{\text {stat }}\right) \times 10^{-12} \mathrm{~cm}^{-2} \mathrm{~s}^{-1}$. The inset shows the flux variations for the flaring nights in more detail. An exponential function, $e^{\lambda t}$ (dashed line) is used to describe the rise and fall time for flux variations giving $\lambda_{\text {rise }}=0.86\left(\chi^{2} /\right.$ dof $\left.=3.7 / 2\right)$ and $\lambda_{\text {fall }}=-0.47\left(\chi^{2} /\right.$ dof $\left.=7.2 / 6\right)$, respectively. The characteristic flux doubling time, $t_{\text {var }}$ is estimated to be on the order of $\sim 1$ day.

(A color version of this figure is available in the online journal.)

Figure 3 shows the light curve of 1ES 1218+304 for the 20082009 season (binned by night). The average integral photon flux above $200 \mathrm{GeV}$ is $\Phi(E>200 \mathrm{GeV})=\left(18.4 \pm 0.9_{\text {stat }}\right) \times$ $10^{-12} \mathrm{~cm}^{-2} \mathrm{~s}^{-1}$ corresponding to $\sim 7 \%$ of the Crab Nebula flux above the same threshold. The light curve from 1ES 1218+304 shows strong nightly flux variations between 2009 January 25 and 2009 February 5. The highest $\gamma$-ray flux is observed on MJD 54861 (2009 January 30) and is 20\% of the Crab Nebula flux. The inset within Figure 3 shows the light curve above $200 \mathrm{GeV}$ for nights with the highest flux. A fit of a constant to the emission during the entire season yields a $\chi^{2} /$ dof of $106 / 21$ (Figure 3, dashed dotted line), corresponding to a very small $\chi^{2}$ probability $\left(\sim 10^{-13}\right)$. The data taken during the active period in January/February of 2009 indicate variability on a timescale of days.

In order to investigate possible changes to the spectral shape during the flaring activity, the total data set is divided into highstate and low-state samples. The two nights with the highest flux during flaring activity (January 30 and 31) are considered the high state, while the remaining nights are considered the low state. Both the high-state and low-state spectra are well described by a power law, and Table 2 summarizes the spectral results. We do not see any evidence for a change in the spectral index during the increase in the absolute flux level from 1ES $1218+304$.

\section{DISCUSSION AND CONCLUSIONS}

A prominent feature in the emission properties of blazars is the observed variability on timescales ranging from minutes to years. In 2006, Suzaku detected a prominent X-ray flare from 1ES 1218+304 revealing a hard-lag variation in the X-rays (Sato et al. 2008). While 1ES 1218+304 was flagged as variable in the Fermi bright source catalog (Abdo et al. 2009b), no variability
Table 2

High-state and the Low-state Data ${ }^{\text {a }}$

\begin{tabular}{lccc}
\hline \hline Spectrum & $\begin{array}{c}\text { Live Time } \\
\text { (minute) }\end{array}$ & $f_{0}$ & $\Gamma$ \\
\hline High & 195 & $19.4 \pm 2.3$ & $3.01 \pm 0.17$ \\
Low & 1436 & $10.0 \pm 0.7$ & $3.12 \pm 0.10$ \\
\hline Avg & 1631 & $11.5 \pm 0.7$ & $3.07 \pm 0.09$ \\
\hline
\end{tabular}

Note.

${ }^{a}$ The high-state and low-state data (see the text for complete description) are well described by a power-law fit of the form $\frac{d N}{d E}=f_{0} \cdot\left(\frac{E}{0.5 \mathrm{TeV}}\right)^{-\Gamma}\left(10^{-12} \mathrm{~cm}^{-2} \mathrm{~s}^{-1} \mathrm{TeV}^{-1}\right)$. We note that changes to the VHE photon index during the flaring activity are statistically insignificant.

was detected in the Fermi light curve (Abdo et al. 2009a). The increased flux measured by VERITAS is the first significant observation of flaring activity in the VHE emission from 1ES $1218+304$. Among the other hard-spectrum VHE blazars (1ES 1101-232, 1ES 0347-121, 1ES 0229+200, 1ES1218+304, RGB J0710+591) measured to date, 1ES 1218+304 is the first clear example of significant VHE variation from a baseline flux. Consequently, the strong flare will challenge existing spectral models of 1ES $1218+304$ that assume a constant VHE emission from the source (see, for example, Rüger et al. 2010).

It is generally assumed that the VHE emission from blazars is produced in the relativistic jet where the highly collimated and Doppler-boosted emission is responsible for the high luminosities and short variability timescales. The size of the emission region, $R$, is constrained by the causality argument to $R \leqslant c t_{\mathrm{var}} \delta /(1+z)$, where $\delta$ is the relativistic Doppler factor and $t_{\mathrm{var}}$ is the observed $\gamma$-ray variability timescale. An estimate 
of the flux-doubling time ( $t_{\mathrm{var}} \lesssim 1$ day; see Figure 3 ) limits the size of the emission region to $R \delta^{-1} \leqslant 2.19 \times 10^{15} \mathrm{~cm} \sim$ $0.71 \times 10^{-3} \mathrm{pc}$. The Doppler factor $(\delta \sim 20)$ typically derived for blazars (Marscher 2006) implies that $R \lesssim 0.01$ pc.

While the hard emission spectrum during the quiet state is consistent with the CMB-inverse-Compton interpretation, the requirement for a large emission region $(\sim$ pc scale $)$ predicted by the model is excluded by the $R \lesssim 0.01$ pc constraint imposed by the variability. Therefore, the flaring behavior from 1ES $1218+304$ implies that the CMB-inverse-Compton model of emission in the kiloparsec-scale extended jet is unlikely to be the sole explanation for the extreme hardness in the intrinsic spectrum of 1ES $1218+304$.

Although our observations of 1ES 1218+304 are consistent with a baseline level of VHE emission that could have its origin in an extended emission region, the variability described here indicates that comparable flux with a comparably hard spectrum is emitted from the compact regions of this source. Further observations of 1ES $1218+304$ are required to determine if the hard-spectrum emission can be explained as originating from the kiloparsec extended jet via the CMB-inverse-Compton model. Consequently, long-term VHE monitoring with ground-based $\gamma$-ray telescopes may be crucial to unraveling the emission mechanism in distant the hard-spectrum VHE blazars.

This research is supported by grants from the U.S. Department of Energy, the U.S. National Science Foundation, the Smithsonian Institution, by NSERC in Canada, by STFC in the U.K. and by Science Foundation Ireland. We acknowledge the excellent work of the technical support staff at the FLWO and the collaborating institutions in the construction and operation of the instrument.

Facilities: VERITAS

\section{REFERENCES}

Abdo, A. A., et al. 2009a, ApJ, 707, 1310 Abdo, A. A., et al. 2009b, ApJS, 183, 46 Acciari, V. A., et al. 2008a, ApJ, 684, L73 Acciari, V. A., et al. 2008b, ApJ, 693, L104 Acciari, V., et al. 2009a, ApJ, 695, 1370

Acciari, V., et al. 2009b, ApJ, 707, 612

Aharonian, F. 2000, New Astron., 5, 377

Aharonian, F., et al. 2006a, Nature, 440, 1018

Aharonian, F., et al. 2006b, A\&A, 457, 899

Aharonian, F., et al. 2007a, A\&A, 475, L9

Aharonian, F., et al. 2007b, A\&A, 473, L25

Aharonian, F., et al. 2008, MNRAS, 387, 1206

Albert, J., et al. 2006, ApJ, 642, L119

Albert, J., et al. 2007, ApJ, 666, L17

Berge, D., Funk, S., \& Hinton, J. 2007, A\&A, 466, 1219

Bloom, S. D., \& Marscher, A. P. 1996, ApJ, 461, 657

Böttcher, M., Dermer, C., \& Finke, J. 2008, ApJ, 679, L9

Hillas, M. 1985, Proc. 19th ICRC (La Jolla), 3, 445

Holder, J., et al. 2006, Astropart. Phys., 25, 391

Katarzyński, K., et al. 2006, MNRAS, 368, L52

Krennrich, F., Dwek, E., \& Imran, A. 2008, ApJ, 689, L23

Levenson, L., \& Wright, E. 2008, ApJ, 683, 585

Li, T., \& Ma, Y. 1983, ApJ, 272, 317

Malkov, M., \& Drury, O’C. 2001, Rep. Prog. Phys., 64, 429

Mannheim, K., \& Biermann, P. L. 1992, A\&A, 253, L21

Maraschi, L., Ghisellini, G., \& Celotti, A. 1992, ApJ, 397, L5

Marscher, A. 2006, in AIP Conf. Proc. 856, Relativistic Jets: The Common Physics of AGN, Microquasars, and Gamma-Ray Bursts, ed. P. A. Hughes \& J. N. Bregman (Melville, NY: AIP), 1

Ong, R. A., et al. 2009, ATel, 2098, 1

Perkins, J., et al. 2009, Proc. 31st ICRC (Lodz), arXiv:0907.4978

Rüger, M., Spanier, F., \& Mannheim, K. 2010, MNRAS, 401, 973

Sánchez-Conde, M., et al. 2009, Phys. Rev. D, 79, 1235

Sato, R., et al. 2008, ApJ, 680, L9

Stecker, F., et al. 2007, ApJ, 667, L29

Tavecchio, F., et al. 2007, ApJ, 665, 980

Teshima, M., et al. 2008, ATel, 1500, 1

Urry, C., \& Padovani, P. 1995, PASP, 107, 803 